\section{Mudanças no padrão epidemiológico da malária em área rural do médio Rio Negro, Amazônia brasileira: análise retrospectiva}

\author{
Changes in the epidemiological pattern of malaria \\ in a rural area of the middle Rio Negro, Brazilian \\ Amazon: a retrospective analysis
}

\author{
I Instituto Oswaldo Cruz, \\ Fundação Oswaldo Cruz, \\ Rio de Janeiro, Brasil. \\ Correspondência \\ J. R. Coura \\ Departamento de \\ Medicina Tropical, \\ Instituto Oswaldo Cruz, \\ Fundação Oswaldo Cruz. \\ Av. Brasil 4365, \\ Rio de Janeiro, $R J$ \\ 21045-900, Brasil. \\ coura@ioc.fiocruz.br
}

\begin{abstract}
A retrospective study on reported malaria cases in the municipality (county) of Barcelos, Amazonas State, Brazil, was performed from 1992 to 2004, emphasizing the high endemic area along the Padauiri, an affluent of the Rio Negro. 16,795 cases were reported, 10,318 (61.4\%) from the rural area and 6,477 (38.6\%) from the urban area. Mean annual parasite index for the period was 136.7 per 1,000 inhabitants in the urban area and 613.6 per 1,000 in the rural area of Barcelos and 708.9 per 1,000 in the Padauiri area. In the latter area, two periods were considered: one epidemic, from 1992 to 1998, and the other post-epidemic, from 1999 to 2004. Comparing the two periods, the malelfemale ratio changed from 1.8 to 1.14, mean patient age from 17.9 to 14.8, proportion of Plasmodium falciparum cases from $51.9 \%$ to $23.7 \%$, proportion of slides with low $\mathrm{P}$. falciparum parasite density from $35.3 \%$ to $44.9 \%$, and proportion of $\mathrm{P}$. vivax from $24 \%$ to $35 \%$ (all these differences were statistically significant, with $p<$ 0.05). The changes in the epidemiological pattern of malaria in the Padauiri area will be further elucidated through prospective studies.
\end{abstract}

Malaria; Health Profile; Amazonian Ecosystem
Martha Cecilia Suárez-Mutis ${ }^{1}$

José Rodrigues Coura ${ }^{1}$

\section{Introdução}

Apesar dos esforços dos programas de controle, a malária continua sendo uma endemia em ascensão no Brasil. O Estado do Amazonas vem apresentando um aumento no número de casos nos últimos três anos e é necessário elucidar a causa deste fenômeno. O Município de Barcelos, médio Rio Negro, é um dos municípios que desde anos atrás tem sido considerado como crítico devido à alta incidência parasitaria anual (IPA), que tem valores acima de 200 por mil habitantes 1. O Rio Padauiri, na fronteira entre os municípios de Barcelos e Santa Isabel do Rio Negro, no Estado do Amazonas, caracteriza-se pela presença de trabalhadores extrativistas de piaçaba, que se deslocam freqüentemente de uma área para outra do rio e que desde muito tempo tem sido considerada uma área altamente endêmica para malária, cuja epidemiologia não tem sido totalmente esclarecida. Embora o sistema de saúde do município faz vários anos realize atividades de controle baseadas no diagnóstico e tratamento, assim como medidas antivetoriais, a malária nesta área permanece sem controle, enfatizando-se, por conseguinte, a necessidade de se entender melhor as características epidemiológicas e a dinâmica de transmissão da infecção neste local.

Com o objetivo de estabelecer o perfil epidemiológico da malária no Rio Padauiri, uma das áreas de maior transmissão do Município de 
Barcelos, foi realizada uma análise retrospectiva dos casos dos últimos 13 anos (1992-2004).

\section{Materiais e métodos}

\section{Área de estudo}

O Município de Barcelos está situado no médio Rio Negro no norte do Estado do Amazonas. A sede do município está localizada a $0^{\circ} 58^{\prime} 1$ ” latitude norte e $62^{\circ} 56^{\prime}$ longitude oeste. No Município de Barcelos vivem 24.197 pessoas, sendo 7.954 moradoras na área urbana $(32,8 \%$ do total da população) e 16.243 na rural 2. Tem uma área de $121.760 \mathrm{~km}^{2}$, o maior município do Brasil, com uma floresta tropical úmida com temperatura entre $20^{\circ}$ e $38^{\circ} \mathrm{C}$ e um índice pluviométrico médio anual de 2.286,2 milímetros, caracterizada pela sazonalidade. As chuvas iniciam-se no mês de março e abril, e as enchentes ocorrem nos meses de junho até agosto. No mês de outubro, o nível dos rios começa a diminuir; o período menos chuvoso vai de outubro até março e os meses mais secos são de janeiro a março, quando é mais intenso o calor e o nível das águas alcança sua cota mínima. A umidade relativa do ar mantém uma média anual de $83 \%$ alterando-se com as mudanças de temperatura no decurso do dia alcançando durante a noite uma saturação de quase $100 \%$.

Administrativamente o programa de controle da malária, subdividiu o município em seis micro-regiões: (1) Área Urbana, (2) Rios Aracá, Demini e Curuduri, (3) Rio Padauiri, (4) Rios Unini e Jaú, (5) Rio Quinini, (6) Ríos Negro e Jufariz. O Rio Padauiri é um afluente da margem esquerda do Rio Negro e é o limite entre os municípios de Barcelos e Santa Isabel do Rio Negro. A área exata do presente estudo é o Rio Padauiri, desde a Cachoeira da Aliança até sua desembocadura no Rio Negro, compreendendo um total de $124 \mathrm{~km}$. A Cachoeira da Aliança é um acidente geográfico no rio, acima da qual existem terras indígenas Yanomámi e marca o limite norte com o Município de Santa Isabel do Rio Negro. Nesta área específica, cinco pequenas localidades são encontradas: Tapera $\left(00^{\circ} 11^{\prime} 41,3^{\prime \prime}\right.$, $\left.64^{\circ} 04^{\prime} 42^{\prime \prime O}\right)$, Acú-Acú $\left(00^{\circ} 06^{\prime} 24^{\prime \prime} \mathrm{S}, 64^{\circ} 01^{\prime} 45^{\prime \prime} \mathrm{O}\right)$, Acuquaia $\left(00^{\circ} 13^{\prime} 15^{\prime \prime} \mathrm{N}\right.$, $\left.63^{\circ} 59^{\prime} 24^{\prime \prime} \mathrm{O}\right)$, Ararinha $\left(00^{\circ} 29^{\prime} 41^{\prime \prime} \mathrm{N}, 64^{\circ} 03^{\prime} 21^{\prime \prime} \mathrm{O}\right)$ e Ararão (00³0'19”'N, 6403'29”O). Existem também outros lugares habitados ao longo do Rio Padauiri denominados sítios, mas têm poucos habitantes.

A economia local gira em torno das atividades do extrativismo fundamentalmente da piaçaba (uma fibra vegetal utilizada para fazer os tetos das casas e confecção de vassouras e cor- das) cuja fibra é extraída de palmeiras (Leopoldinia piassaba) que se localizam em colocações nas cabeceiras dos rios da margem esquerda do Rio Negro. Os piaçabeiros estão subordinados ao regime de aviamento (forma de recrutamento da força de trabalho por meio do fornecimento de mercadorias - alimentação, roupas, combustível etc. - em adiantamento, para o sustento do trabalhador enquanto realiza esta atividade. Os preços das mercadorias cobrados pelos "patrões" são muito altos e, portanto, os trabalhadores estão sempre endividados). Para esta atividade as pessoas se deslocam continuamente ao longo do rio determinando uma ampla mobilidade espacial no nível local. As vias de transporte disponíveis nesta área são exclusivamente os rios, conseqüentemente os únicos meios de transporte são pequenas embarcações. Em Barcelos existe uma Secretaria Municipal de Saúde e no momento do estudo a Fundação Nacional de Saúde (FUNASA) era a responsável pelas ações de controle da malária, atualmente sob a responsabilidade daquela Secretaria.

\section{Metodologia}

Trata-se de um estudo retrospectivo. Foram utilizados dados secundários referentes ao período de 1992 até 2004 (informação disponível), usando como fonte de informação os livros de notificação de caso da FUNASA do Município de Barcelos obtidos na sede municipal e a ficha de notificação do Ministério de Saúde. Foram definidos como caso todos os pacientes com sintomas clínicos de malária confirmados pela gota espessa positiva segundo parâmetros da FUNASA. Considerando que em estudos prévios do nosso grupo na área com outras doenças havia sido encontrada uma grave situação epidemiológica de malária no Rio Padauiri, decidimos aprofundar nossa pesquisa neste lugar. Foram utilizadas as seguintes variáveis: (1) espacial (município, área urbano-rural, microrregiões e localidades do Rio Padauiri), (2) temporal (ano, mês, sazonalidade), (3) demográficas (sexo e faixa etária), e (4) parasitológicas (espécie e densidade parasitária). Foi considerada baixa densidade parasitária quando as gotas espessas tinham menos de 50 parasitos por campo microscópico.

\section{Análise estatística}

Todos os dados foram armazenados e analisados usando a versão 6.0 do programa Epi Info (Centers for Disease Control and Prevention, Atlanta, Estados Unidos). Foi usado o teste do qui-quadrado $\left(\chi^{2}\right)$ para analisar as diferenças nas proporções de casos por sexo, idade, estação do ano 
e espécie parasitaria. A estatística T foi utilizada para comparação de médias. Um $\mathrm{p}$ valor $<0,05$ foi considerado estatisticamente significativo.

\section{Aspectos éticos}

Esta pesquisa faz parte de um projeto maior denominado Estudo da Malária em Áreas de Baixa e Alta Morbidade no Médio e Alto Rio Negro, Estado do Amazonas e Novas Estratégias de Controle e foi aprovado pelo Comitê de Ética da Fundação Oswaldo Cruz sob o número 157/02.

\section{Resultados}

\section{A malária na população do}

Município de Barcelos

Desde 1992 até o ano 2004 foram notificados 16.795 casos de malária no Município de Barcelos; dentre eles, 10.318 (61,43\%) procediam da área rural e 6.477 (38,57\%) da área urbana. A média de notificações anuais durante o período foi de 1.292 casos, 498 (38,54\%) casos na área urbana e 794 (61,46\%) na área rural. Os maiores números de casos foram notificados nos anos de 1995, 2003, 2004 e 1998 (15,2, 12,9, 12,8 e 11\% das notificações, respectivamente). Os anos em que o número absoluto de casos urbanos superou os da área rural foram 1994 e 2004 quando a área urbana contribuiu com $58,7 \%$ e a rural com $51,4 \%$ dos casos, respectivamente (Tabela 1).

A área do Rio Negro contribuiu com 50,4\% (5.203/10.318) dos casos da malária da área rural no período. Esta área compreende comunidades localizadas no Rio Negro, muitas das quais ficam muito próximas da área urbana do Município de Barcelos. No Rio Aracá também ocorre grande número de casos de malária, responsável por $16 \%(1.649 / 10.318)$ dos casos seguido pelo Rio Padauiri (13,6\%, 1.399/10.318) e o Rio Unini $(8,5 \%, 882 / 10.318)$. Outros correspondem a casos importados para o Município de Barcelos procedentes tanto de áreas urbanas e rurais de outros municípios e foi responsável por 8,2\% das notificações. O Rio Quinini contribuiu com 3,3\% dos casos (335/10.318) (Tabela 1). Apesar de existir claramente uma maior ocorrência da malária em comunidades de alguns rios, o número de casos varia de mês em mês e de ano em ano sem que se possa evidenciar um padrão determinado por microrregiões no município como um todo ao longo dos anos.

Durante o ano 2004, a IPA foi 136,7 por 1.000 habitantes na área urbana e de 613,6 na área rural. Ao estratificarmos por rios na área rural, a IPA foi de 708,9 por 1.000 habitantes no Rio Padauiri,
644,4 no Rio Unini, 622 no Rio Negro, 514,3 no Rio Quinini e 457,6 no Rio Aracá.

\section{Epidemiologia da malária no Rio Padauiri}

Durante os 13 anos do estudo, foram notificados um total de 1.399 casos de malária procedentes do Rio Padauiri contribuindo com 13,6\% do total dos casos da área rural, sendo que o ano de 1997 contribuiu com $30,4 \%$ do total de casos da área rural. No Rio Padauiri observou-se um incremento nas notificações de malária em 1993 (175 casos), 1998 (183 casos) e 2003 (135 casos) em relação aos demais anos deste estudo. Na Figura 1, observa-se claramente que a epidemiologia da malária nesta área pode ser dividida em dois períodos: o primeiro que denominaremos de epidêmico, inclui desde o inicio das notificações até o ano de 1998, período durante o qual o Rio Padauiri contribui com 14,9\% das notificações da área rural (863/5.796) e o Plasmodium falciparum foi o parasito predominante. Um segundo período que denominaremos de pós-epidêmico que compreende de 1999 a 2004 no qual essa área contribuiu com $11,9 \%$ do total das notificações (536/4.522) com predomínio do Plasmodium vivax. Essas diferenças foram estatisticamente significativas $(\mathrm{p}<0,05)$.

\section{Lugar e incidência da malária}

Para analisarmos a epidemiologia da malária em comunidades do Rio Padauiri, a área foi classificada em cinco setores baseados nas características geográficas e populacionais. O primeiro setor corresponde à área próxima da desembocadura do Rio Padauiri no Rio Negro, e se caracteriza porque se encontra a maior localidade (Tapera) que recebe pessoas procedentes tanto do Rio Padauiri quanto do Rio Preto em trânsito para Barcelos. Nessa área a IPA média para malária foi de 133,5 por mil habitantes. No total, foram notificados $66,4 \%(83 / 125)$ de casos de malária por $P$. vivax e 33,6\% (42/125) de casos por P.falciparum. O segundo setor representa as comunidades ao redor de Acú-Acú. Com uma IPA média de 292,7 por mil habitantes, ocorreram nesse setor $76,3 \%$ (119/156) casos de malária por P. vivax e 22,4\% (35/156) casos de malária por P. falciparum. O terceiro setor compreende as comunidades ao redor de Acuquaia com uma IPA de 501,3 por mil habitantes, dos quais 58,1\% (227/391) foram devidos a $P$. vivax e $41,9 \%(164 / 391)$ a $P$ falciparum. O quarto setor corresponde a localidades próximas à Ararinha, Ararão e Igarapé do Tabaco, cuja IPA média foi de 671,9 por mil habitantes, tendo 53,1\% (297/559) dos casos devidos a $P$. vivax e 46,9\% (262/559) devidos ao P. falciparum. 
Tabela 1

Casos de malária notificados no Município de Barcelos, Amazonas, Brasil, segundo área geográfica de procedência, $1992-2004$.

\begin{tabular}{|c|c|c|c|c|c|c|c|c|c|c|c|c|c|c|c|c|c|}
\hline \multirow[t]{3}{*}{ Ano } & \multirow{2}{*}{\multicolumn{2}{|c|}{ Área urbana }} & \multicolumn{14}{|c|}{ Área rural } & \multirow{3}{*}{$\begin{array}{c}\text { Total } \\
\text { município }\end{array}$} \\
\hline & & & \multicolumn{2}{|c|}{ Padauri } & \multicolumn{2}{|c|}{ Aracá } & \multicolumn{2}{|c|}{ Quinini } & \multicolumn{2}{|c|}{ Unini } & \multicolumn{2}{|c|}{ Negro } & \multicolumn{2}{|c|}{ Outros } & \multicolumn{2}{|c|}{ Total rural } & \\
\hline & $\mathrm{n}$ & $\%$ * & $\mathrm{n}$ & $\%$ ** & $\mathrm{n}$ & $\%$ ** & $\mathrm{n}$ & $\%$ ** & $\mathrm{n}$ & $\%$ ** & $\mathrm{n}$ & $\%$ ** & $\mathrm{n}$ & $\%$ ** & $\mathrm{n}$ & $\%$ * & \\
\hline 1992 & 459 & 32,8 & 115 & 12,2 & 39 & 4,2 & 126 & 13,4 & 5 & 0,5 & 468 & 49,8 & 186 & 19,8 & 939 & 67,2 & 1.398 \\
\hline 1993 & 195 & 20,6 & 175 & 23,3 & 55 & 7,3 & 15 & 2,0 & 7 & 0,9 & 378 & 50,3 & 121 & 16,1 & 751 & 79,4 & 946 \\
\hline 1994 & 892 & 58,7 & 58 & 9,2 & 119 & 18,9 & 10 & 1,6 & 13 & 2,1 & 381 & 60,7 & 47 & 7,5 & 628 & 41,3 & 1.520 \\
\hline 1995 & 1.270 & 49,7 & 80 & 6,2 & 281 & 21,9 & 40 & 3,1 & 12 & 0,9 & 760 & 59,1 & 113 & 8,8 & 1.286 & 50,3 & 2.556 \\
\hline 1996 & 206 & 21,7 & 119 & 16,0 & 169 & 22,7 & 22 & 3,0 & 46 & 6,2 & 351 & 47,1 & 38 & 5,1 & 745 & 78,3 & 951 \\
\hline 1997 & 136 & 23,7 & 133 & 30,4 & 52 & 11,9 & 13 & 3,0 & 41 & 9,4 & 145 & 33,1 & 54 & 12,3 & 438 & 76,3 & 574 \\
\hline 1998 & 837 & 45,3 & 183 & 18,1 & 277 & 27,5 & 16 & 1,6 & 9 & 0,9 & 422 & 41,8 & 102 & 10,1 & 1.009 & 54,7 & 1.846 \\
\hline 1999 & 366 & 32,9 & 122 & 16,4 & 245 & 32,8 & 23 & 3,1 & 35 & 4,7 & 292 & 39,1 & 29 & 3,9 & 746 & 67,1 & 1.112 \\
\hline 2000 & 52 & 8,8 & 66 & 12,3 & 63 & 11,8 & 11 & 2,1 & 199 & 37,1 & 141 & 26,3 & 56 & 10,4 & 536 & 91,2 & 588 \\
\hline 2001 & 23 & 7,1 & 24 & 8,0 & 56 & 18,6 & 3 & 1,0 & 118 & 39,2 & 81 & 26,9 & 19 & 6,3 & 301 & 92,9 & 324 \\
\hline 2002 & 160 & 23,8 & 77 & 15,0 & 59 & 11,5 & 14 & 2,7 & 138 & 27,0 & 204 & 39,8 & 20 & 3,9 & 512 & 76,2 & 672 \\
\hline 2003 & 777 & 36,0 & 135 & 9,8 & 153 & 11,1 & 24 & 1,7 & 105 & 7,6 & 940 & 68,0 & 26 & 1,9 & 1.383 & 64,0 & 2.160 \\
\hline 2004 & 1.104 & 51,4 & 112 & 10,7 & 81 & 7,8 & 18 & 1,7 & 154 & 14,8 & 640 & 61,3 & 39 & 3,7 & 1.044 & 48,6 & 2.148 \\
\hline Total & 6.477 & 38,6 & 1.399 & 8,3 & 1.649 & 9,8 & 335 & 2,0 & 882 & 5,3 & 5.203 & 31,0 & 850 & 5,16 & 10.318 & 61,4 & 16.795 \\
\hline$\%$ rural & & & & 13,6 & & 16,0 & & 3,2 & & 8,5 & & 50,4 & & 8,2 & & 100,0 & \\
\hline
\end{tabular}

* Percentual do total do município;

** Percentual do total da área rural.

Figura 1

Número anual de casos de malária no Rio Padauiri registrados na Fundação Nacional de Saúde, no Município de Barcelos, Amazonas, Brasil, e o percentual de casos gerados por $P$. falciparum baseado no exame da gota espessa, obtido durante a rotina de pacientes febris, de 1992 até 2004.

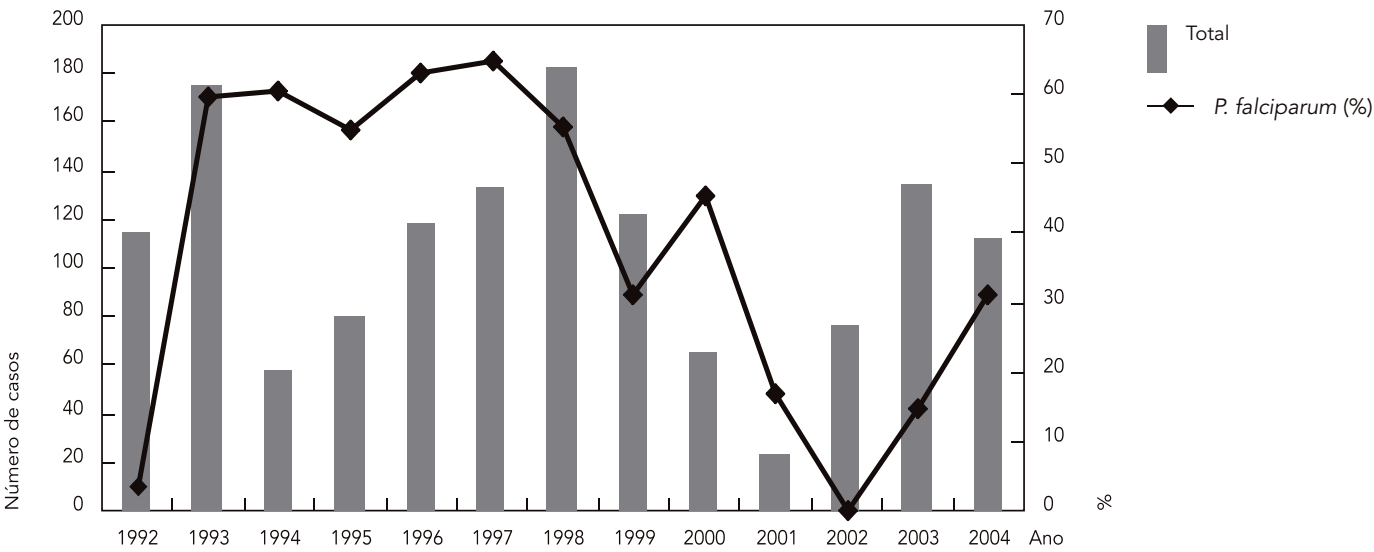


No quinto setor estão as comunidades acima do Igarapé do Tabaco, incluindo alguns sítios perto da Cachoeira da Aliança e algumas notificações do posto da Fundação Nacional do Índio sem ser população Yanomámi. A IPA neste quinto setor foi de 903,8 por mil habitantes; 46,8\% (44/94) casos foram por P. vivax e 53,2\% (50/94) por $P$. falciparum. Ao observar os casos de malária registrados tendo em conta as localidades mais próximas aos grandes piaçabais localizados nos setores 3, 4 e 5, encontra-se que 77,1\% dos casos registrados no rio Padauiri durante o período analisado ocorreram nas áreas de extrativismo de piaçaba enquanto $22,9 \%$ foram registrados longe das áreas de piaçabais. A chance de que os casos de P. falciparum acontecessem nas áreas de piaçabais foi 2,13 vezes maior quando comparada às áreas mais distantes dos piaçabais (IC: 1,60-2,84; $\mathrm{p}<0$,05) (Figura 2).

\section{Distribuição por gênero e grupo etário}

Durante o período de estudo houve um predomínio dos casos de malária em crianças menores de 15 anos (52,7\%, 737/1.399), sendo 48,8\% deles (360/737) em menores de 5 anos. Desde 1992 até 1998 o maior número de casos foi registrado entre os maiores de 15 anos (50,9\%, 439/863), mas a partir de 1999 até 2004 o predomínio das notificações foi entre os menores de 15 anos (58,4\%, 313/536). A média de idade entre os indivíduos que adoeceram por malária no período epidêmico foi de 17,9, e a média de idade depois de 1998 foi de 14,8 (Figura 3).

No período de 1992 até2004, 40,1\% (561/1399) mulheres e 59,9\% (838/1.399) homens adoeceram por malária no Rio Padauiri. Antes de 1998, os maiores números de casos notificados foram em homens $(64,1 \%, 553 / 863)$, se comparados com as mulheres (35,9\%, 310/863), enquanto no período de 1999 até 2004 , apesar de o maior número de casos registrados entre os homens ainda permanecer, o percentual diminuiu em relação ao gênero feminino (homens 53,2\%, 285/536; mulheres, 46,8\%, 251/536). A proporção de casos de homens/mulheres no total durante o período foi de 1,49 (838/561), e a proporção no período epidêmico foi de 1,78 (553/310) e depois de 1998 foi de 1,14 (285/251). Ao comparar tais proporções, observa-se que há uma diminuição na proporção de homens/mulheres nos anos pós-epidêmicos comparados com a proporção existente nos anos epidêmicos. Essas diferenças foram estatisticamente significativas $(\mathrm{p}<0,05)$.

\section{Espécie parasitária}

Durante o período, 41,1\% (575/1.399) dos casos diagnosticados de malária corresponderam à infecção pelo $P$. falciparum, 57,8\% (809/1.399) ao $P$. vivax e 1\% (14/1.399) à infecção mista. De 1992 até 1998, o P. falciparum foi o parasito predominante, com 51,9\% dos casos (448/863); depois da epidemia de 1998, o P. vivax passou a ser a espécie parasitária predominante $(75,2 \%, 403 / 536)$. Todas essas diferenças foram estatisticamente significativas $(\mathrm{p}<0,05)$ (Figura 1 e Tabela 2$)$.

Um total de 737 crianças menores de 15 anos teve malária; $247 / 737$ (33,5\%) padeceram de infecção por P.falciparum e 489/737 (66,3\%) por P.vivax. No período epidêmico, 183/424 (43,2\%) tiveram P. falciparum e 240/424 (56,6\%) P. vivax. No período pós-epidêmico 64/313 (20,4\%) tiveram malária por $P$ falciparum e 249/313 (79,6\%) por $P$. vivax.

Também foram realizadas análises da densidade parasitária segundo espécie. Durante todo o período, 29,4\% (238/809) das lâminas dadas como positivas para P. vivax tinham uma baixa densidade parasitária; 24\% (97/406) das lâminas tiveram uma baixa densidade parasitária no período epidêmico e 35\% (141/403) no período pósepidêmico. Para o caso do P. falciparum, 37,4\% $(215 / 575)$ das gotas espessas tiveram uma baixa parasitemia, sendo $35,3 \%$ (158/448) no período epidêmico e 44,9\% (57/127) no pós-epidêmico. Todas essas diferenças foram estatisticamente significativas $(\mathrm{p}<0,05)$.

\section{Discussão}

O presente trabalho baseia-se em dados secundários, que, em análises epidemiológicas, podem dar origem a distorções pela presença de subnotificação de casos que pode acontecer em áreas esparsas como a Amazônia. No entanto, apesar das limitações do sistema de notificação oficial da malária, sua análise seqüencial em período superior a dez anos (ano a ano), como é o caso deste trabalho, em áreas nas quais se tem pouco conhecimento desta endemia, permite obter informações sobre a heterogeneidade e variação de sua situação em diferentes lugares do Brasil. Sem dúvida, a malária é um problema "glolocal" (termo sociológico usado para se referir aos problemas globais que têm características locais.) que tem especificidades segundo as condições ecológicas, sanitárias, sociais, políticas, e culturais de cada lugar. Tal ponto é importante não somente para a tomada de decisões no concernente aos programas de controle, mas também para planejar novas pesquisas que permitam entender situações pouco definidas. 
Incidência parasitária anual média da malária no Rio Padauiri no período entre 1992 e 2004 e distribuição das espécies parasitárias (subdivisão por setores) no Rio Padauiri, limite entre os municípios de Barcelos e Santa Isabel do Rio Negro, Estado do Amazonas, Brasil.

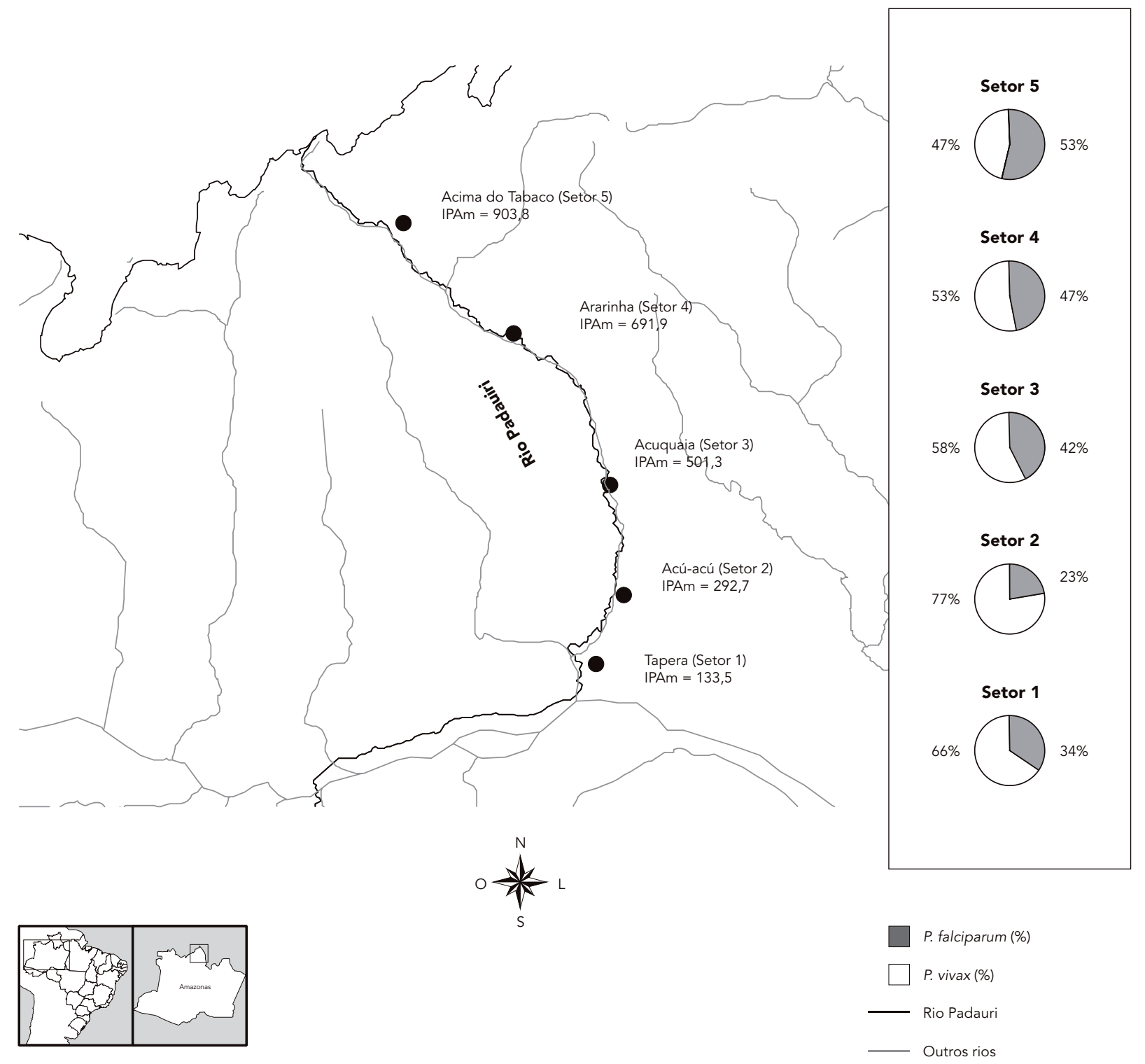

Barcelos é uma área estudada desde o inicio do século XX por diferentes cientistas; contudo, estudos focais em relação à epidemiologia da malária no momento atual são escassos. As informações obtidas neste estudo permitiram realizar algumas observações importantes. Primeiro, ao contrário do que ocorreu no Amazonas como um todo, com diminuição de $12,7 \%$ dos casos de ma- lária, comparando 1999 com 2004, no Município de Barcelos houve um incremento de 93,2\% no mesmo período em razão de um aumento na incidência a expensas fundamentalmente dos casos da área urbana $(201,6 \%)$, pois na área rural o incremento foi de apenas 39,9\%. Na Amazônia brasileira, a maioria de casos ocorre em áreas rurais. Porém, nos anos 2003 e 2004, cidades co- 
Casos de malária por grupo de idade no período epidêmico, entre 1992 e 1998, (média de idade = 17,9) e no período pósepidêmico, entre 1999 e 2004 (média de idade = 14,8).

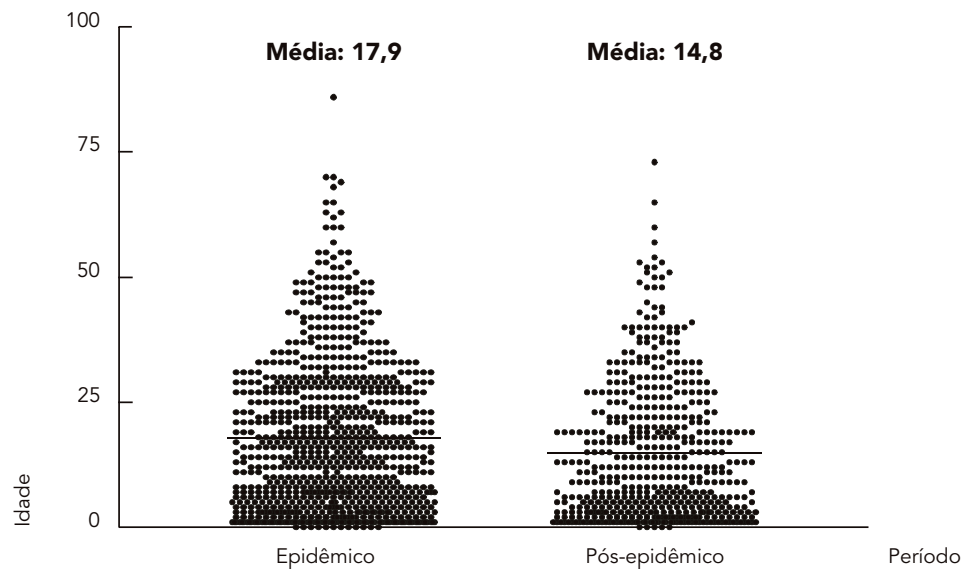

mo Manaus e Porto Velho concentraram 26,9\% e 22,9\% dos casos de malária na região Amazônica, respectivamente ${ }^{3}$. Já Cevallos 4 havia apontado o incremento da malária no Município de Barcelos por conta dos casos urbanos.

Segundo a aproximação do MacDonald 5, a endemicidade da malária pode ser categorizada em três tipos: a malária estável, que ocorre em áreas nas quais as pessoas estão constantemente expostas, assegurando o desenvolvimento de imunidade contra a doença, exceto para as crianças de baixa idade, que em pouco tempo vão ter sua primeira experiência com o parasito. Nessas áreas de malária estável, os adultos são normalmente assintomáticos ou oligossintomáticos e apresentam uma baixa parasitemia. A malária é mais freqüente nas crianças e aqueles menores de dois anos sofrem risco muito alto de adoecer e morrer por malária. Essas situações ocorrem comumente na África Sub-Saariana, em alguns locais do Sudeste Asiático e da América Latina. A malária instável acontece em lugares em que as pessoas estão submetidas a uma transmissão mais ou menos permanente, entretanto sob circunstâncias nas quais há largas flutuações nas taxas de inoculação do parasito e os indivíduos podem receber inóculos em intervalos anuais ou de vários anos. Os adultos são normalmente acometidos e as crianças menores de cinco anos são poupadas. O terceiro tipo, a malária epidêmica, que na realidade é uma forma extrema da malária instável com epidemias quando as popula- ções não imunes estão sujeitas a um incremento nas taxas de transmissão. Os últimos dois tipos são os predominantes nas Américas 6 .

Apesar da heterogeneidade desta endemia, em geral, considera-se que na Amazônia brasileira a transmissão e a endemicidade da malária são de média e baixa intensidade $3,7,8$, sendo consideradas áreas de transmissão instável 9. A incidência da malária varia de mês para mês e de ano para ano. Apresentam-se surtos epidêmicos estacionais que podem chegar a ser de grandes proporções quando as condições climáticas são ótimas, com grandes variações que decorrem por causas inaparentes. Têm sido registrados ciclos com períodos de 5 a 8 anos. O grau de imunidade da população dessas áreas é muito variável. Nesses lugares, a intensidade da transmissão não permite que as pessoas desenvolvam um alto grau de imunidade naturalmente adquirida e, portanto, não chega a ser protetora 10 . Embora as epidemias atinjam grande parte das pessoas, a imunidade pode ser muito baixa. As crianças freqüentemente escapam da infecção 5,6,11.

Na Amazônia brasileira, 68\% das notificações de malária ocorrem entre adultos maiores de 15 anos. No Rio Padauiri, observa-se, particularmente, uma diferença na notificação por grupo etário; no período pós-epidêmico, 58,4\% dos casos ocorreram em crianças com menos de 15 anos. Também foi demonstrado que está existindo uma mudança no padrão epidemiológico, pois a média de idade tem mudado significativamente 
Comparativo dos casos de malária no período epidêmico e pós-epidêmico segundo gênero, grupo de idade, espécie parasitária e densidade da infecção.

\begin{tabular}{|c|c|c|c|c|c|c|c|c|}
\hline & \multicolumn{2}{|c|}{ Total 1992-2004 } & \multicolumn{2}{|c|}{ Período epidêmico } & \multicolumn{2}{|c|}{ Período pós-epidêmico } & \multirow[t]{2}{*}{$\chi^{2}$} & \multirow[t]{2}{*}{$p$ valor } \\
\hline & $\mathbf{n}$ & $\%$ & $\mathbf{n}$ & $\%$ & $\mathbf{n}$ & $\%$ & & \\
\hline \multicolumn{9}{|l|}{ Casos de malária } \\
\hline no município & 16.795 & & 9.791 & 58,3 & 7.004 & 41,7 & 921,91 & $<0,05$ \\
\hline \multicolumn{9}{|l|}{ Casos de malária } \\
\hline na área rural & 10.318 & 61,3 & 5.796 & 56,1 & 4.522 & 43,8 & 312,15 & $<0,05$ \\
\hline \multicolumn{9}{|l|}{ Casos de malária } \\
\hline no Rio Padauri & 1.399 & 13,6 & 863 & 61,7 & 536 & 38,3 & 152,86 & $<0,05$ \\
\hline \multicolumn{9}{|l|}{ \% de contribuição } \\
\hline dos casos na área rural & 1.399 & 13,6 & $863 / 5.796$ & 14,9 & $536 / 4.522$ & 11,9 & 17,12 & $<0,05$ \\
\hline \multicolumn{9}{|l|}{ Casos de malária } \\
\hline \multicolumn{9}{|l|}{ segundo gênero } \\
\hline Mulheres & 561 & 40,1 & $310 / 863$ & 35,9 & $251 / 536$ & 46,8 & 16,8 & $<0,05$ \\
\hline Homens & 838 & 59,9 & $553 / 863$ & 64,1 & $258 / 536$ & 53,2 & 16,4 & $<0,05$ \\
\hline \multicolumn{9}{|l|}{ Proporção } \\
\hline homens/mulheres & 1,49 & & 1,78 & & 1,14 & & 16,29 & $<0,05$ \\
\hline \multicolumn{9}{|l|}{ Casos de malária } \\
\hline em menores de 15 anos & 737 & 52,7 & $424 / 863$ & 49,1 & $313 / 536$ & 58,4 & 11,83 & $<0,05$ \\
\hline Média de idade & 16,7 & & 17,9 & & 14,8 & & & $<0,05$ \\
\hline \multicolumn{9}{|l|}{ Casos de malária } \\
\hline por P. falciparum & 575 & 41,1 & $448 / 863$ & 51,9 & $127 / 536$ & 23,7 & 108,27 & $<0,05$ \\
\hline \multicolumn{9}{|l|}{ Casos de malária } \\
\hline por P. vivax & 809 & 57,8 & $406 / 863$ & 47,0 & $403 / 536$ & 75,2 & 110,03 & $<0,05$ \\
\hline \multicolumn{9}{|l|}{ Casos de malária } \\
\hline \multicolumn{9}{|l|}{ em menores de 15 anos } \\
\hline P. falciparum & 247 & 33,5 & $183 / 424$ & 43,2 & $64 / 313$ & 20,4 & 41,69 & $<0,05$ \\
\hline P. vivax & 489 & 6,3 & $240 / 424$ & 56,6 & $249 / 313$ & 79,6 & 42,48 & $<0,05$ \\
\hline \multicolumn{9}{|l|}{ Baixa densidade } \\
\hline \multicolumn{9}{|l|}{ parasitária na população } \\
\hline P. falciparum & 215 & 37,4 & $158 / 448$ & 35,3 & $57 / 127$ & 44,9 & 3,91 & $<0,05$ \\
\hline P. vivax & 238 & 29,4 & $97 / 406$ & 24 & $141 / 403$ & 35 & 11,99 & $<0,05$ \\
\hline
\end{tabular}

nos últimos 13 anos, visto que, cada vez mais, crianças novas adoecem por malária, poupando os adultos. Ainda que no inicio do estudo o maior número de casos fosse entre adultos, esse percentual não era tão alto quanto à média nacional, revelando uma situação epidemiológica diferenciada.

Em contraste com os estudos clássicos na Amazônia 3,8,9,12, em uma comunidade ribeirinha em Portochuelo (Rondônia), Camargo et al. 13 encontraram uma maior prevalência de malária em menores de 16 anos, especialmente em crianças menores de um ano. Os adultos maiores de 50 anos foram poupados relativamente, indicando a presença de transmissão intradomiciliar e sugerindo certo grau de resistência adquirida à infecção e/ou uma diminuição na prevalência de sintomas nos adultos. Naquela área a IPA era de 292/1.000 habitantes, sendo uma área de elevado risco de transmissão como no Rio Padauiri. Resultados similares foram encontrados por Marcano et al. 14 na área Yanomámi, na fronteira entre Brasil e Venezuela, em que $95 \%$ e $51,3 \%$ dos casos de malária detectados no alto Orinoco e alto Mucajaí, respectivamente, foram em menores de 16 anos. Os autores mostraram que no alto Orinoco a malária comporta-se como uma área hiperendêmica com transmissão estável em contraposição com o alto Mucajaí, onde a malária é mesoendêmica e instável.

Um estudo posterior realizado em Portochuelo e em Ji-Paraná (Rondônia) mostrou que a média de idade das pessoas que adoeceram por malária foi de 14 anos em Portochuelo e 8 anos em Ji-Paraná. A informação descritiva que conseguimos obter dos dados avaliados no Rio 
Padauiri é similar aos dos estudos mencionados. Alves et al. 15 encontraram a presença de infecção assintomática nessas comunidades (20\% em Portochuelo e 49,5\% em Ji-Paraná) e esta foi mais prevalente que a doença clínica. Marcano et al. 14 também encontraram portadores assintomáticos no alto Orinoco (38,5\%) e no alto $\mathrm{Mu}$ cajai $(4,9 \%)$. Por mais que nesta pesquisa não se pretenda detectar portadores assintomáticos, o perfil epidemiológico encontrado está indicando que no Rio Padauiri o problema pode estar se apresentado e que são necessárias novas pesquisas encaminhadas para esclarecer tal ponto. O fato do aumento no número de lâminas com baixa parasitemia tanto de P. falciparum quanto de $P$. vivax, comparando os dois períodos, pode indicar o inicio do desenvolvimento de imunidade clínica, reforçando ainda mais a hipótese de possíveis portadores assintomáticos de Plasmodium nessa área.

Outro achado importante é a mudança de padrão da doença quanto ao gênero. A malária de áreas instáveis é uma doença que atinge mais os homens adultos. No nosso estudo, encontramos uma diferença nos dois períodos estudados, e a proporção mudou de 1,78 para 1,14. Nossos resultados são similares aos de Camargo et al. 13 nos quais os menores de 16 anos pertencem ao grupo de maior risco independente do sexo.

A distribuição da malária por espécie de parasito no Brasil era similar até 1989 9,16 quando começou a ocorrer uma redução acentuada do P. falciparum e um predomínio crescente do $P$. vivax, que em 1997 era 75,4\% do total. No Rio Padauiri, o $P$. falciparum foi a espécie predominante até a epidemia de 1998. A partir daí o $P$. vivax é o parasito mais notificado, assimilandose aos casos diagnosticados no Brasil. É usual que, quando se iniciam as atividades de controle, seja o $P$ falciparum a primeira espécie a desaparecer de uma área determinada dado que seus gametócitos só começam a aparecer no sangue entre os dias 10-20 da infecção. Assim, quando se realizam intervenções adequadas numa área, rapidamente diminui o número de portadores desses gametócitos. Com o P. vivax, é mais difícil o controle, já que os gametócitos estão circulando no sangue antes do início dos sintomas. O agravamento da situação epidemiológica nos últimos dois anos pode ser em razão de a gerência do programa ter priorizado as ações ao combate ao dengue, diminuindo as atividades de controle de malária nas áreas rurais. Apesar da notória diminuição de casos nos anos 2001 e 2002, a partir do 2003, verifica-se outra vez um incremento dos casos de $P$. falciparum, situação extremamente preocupante, dado que nesta área existe resistência aos fármacos habituais. A mefloquina é o medicamento mais usado pelos ribeirinhos para tratar de sua doença.

No nosso estudo, pode-se observar uma mudança no padrão epidemiológico da malária que atingia inicialmente mais os homens adultos e agora afeta em maior proporção as crianças, com um expressivo aumento de casos entre as mulheres. Deve ser reconsiderada a classificação do tipo de malária no Rio Padauiri. Classificada como de tipo instável, a situação epidemiológica mostra que o risco de malária tem sido persistentemente alto nos últimos 13 anos com mudanças no padrão, assemelhando-a ao de malária estável. Uma explicação potencial da incidência sustentada da transmissão da malária nessa área talvez seja o fato de as infecções acontecerem em clusters, em pequenos subgrupos da população, ou, ainda, que existam portadores assintomáticos não detectados 17. Este ponto deve ser esclarecido mediante estudos prospectivos.

\section{Resumo}

Foi realizado um estudo retrospectivo dos casos de malária ocorridos entre 1992 e 2004 no Município de Barcelos, Amazonas, Brasil, aprofundando as informações existentes na área altamente endêmica do Rio Padauri, afluente do Rio Negro. Foram identificados 16.795 casos de malária, sendo 61,4\% (10.318) da área rural e 38,6\% (6.477) da área urbana, com uma incidência parasitária anual média de 136,7 por mil habitantes na área urbana e 613,6 na área rural. A incidência parasitária anual média no Rio Padauri foi de 708,9/1.000. Nesse rio, foram diferenciados dois períodos epidemiológicos, sendo um denominado epidêmico (1992-1998) e outro pós-epidêmico (19992004). Comparando os dois períodos, a proporção homem/mulher mudou de 1,8 para 1,14, a média de idade dos pacientes passou de 17,9 para 14,8, o percentual de casos por Plasmodium falciparum reduziu-se de 51,9\% para 23,7, e o percentual de lâminas com baixa densidade parasitária de P. falciparum passou de $35,3 \%$ para $44,9 \%$, e as de P. vivax de $24 \%$ para $35 \%$. Todas essas diferenças foram estatisticamente significativas $(p<0,05)$. Tais mudanças no padrão epidemiológico da malária no rio Padauiri devem ser esclarecidas mediante estudos prospectivos.

Malária; Perfil de Saúde; Ecossistema Amazônico 


\section{Referências}

1. Albuquerque BCJ, Suárez-Mutis MC. A malária no Amazonas. In: Iñiguez-Rojas LB, Toledo LM. organizadores. Espaço e doença: um olhar sobre o Amazonas. Atlas de geografia médica. Rio de Janeiro: Editora Fiocruz; 1998. p. II.2.1-10.

2. Instituto Brasileiro de Geografia e Estatística. Dados preliminares do censo 2000: Brasil e Norte. v. 1. Rio de Janeiro: Instituto Brasileiro de Geografia e Estatística; 2001.

3. Secretaria de Vigilância em Saúde, Ministério da Saúde. Situação epidemiológica da malária no Brasil, 2005. http://portal.saude.gov.br/portal/ arquivos/pdf/situacao_epidemio_malaria.pdf (acessado em 10/Nov/2005).

4. Cevallos WT. Estudo do processo de transmissão da malária humana na sede do município de Barcelos, Estado do Amazonas, Brasil [Dissertação do Mestrado]. Rio de Janeiro: Instituto Oswaldo Cruz, Fundação Oswaldo Cruz; 2001.

5. MacDonald G. The epidemiology and control of malaria. London: Oxford University Press; 1957.

6. Carter R, Mendis K. Evolutionary and historical aspects of the burden of malaria. Clin Microbiol Rev 2002; 15:564-94.

7. Loiola CC, Silva CJ, Tauil PL. Controle da malária no Brasil: 1965-2001. Rev Panam Salud Publica 2002; 11:235-44

8. López-Antuñano F. Epidemiology and control of malaria and other arthropod born diseases. Mem Inst Oswaldo Cruz 1992; 87 Suppl 3:105-14.

9. Passos ADC, Fialho RR. Malária: aspectos epidemiológicos e de controle. Rev Soc Bras Med Trop 1998; 31:93-105.

10. Braga EP, Kretti AU. Immune response to sporozoite and blood stage antigens among subjects exposed to malaria in Brasil: a review. Ciênc Cult (São Paulo) 2000; 52:246-52.
11. Rey L. Parasitologia. Parasitos e doenças parasitárias do homem nas Américas e na África. Rio de Janeiro: Editora Guanabara Koogan; 2001.

12. Camargo LMA, Colleto GMD, Ferreira MU, Gurgel SM, Escobar AL, Marques A, et al. Hypoendemic malaria in Rondonia (Brazil, Western Amazon Region): seasonal variation and risk groups in an urban locality. Am J Trop Med Hyg 1996; 55:32-8.

13. Camargo LMA, Noronha E, Salcedo JMV, Dutra AP, Krieger H, Silva LHP, et al. The epidemiology of malaria in Rondonia (Western Amazon Region, Brazil): study of a riverine population. Acta Trop 1999; 72:1-11.

14. Marcano T, Morgado A, Tosta CE, Coura JR. Crosssectional study defines difference in malaria morbidity in two Yanomami communities on Amazonian boundary between Brazil and Venezuela. Mem Inst Oswaldo Cruz 2004; 99:369-76.

15. Alves F, Durlacher RR, Menezes MJ, Krieger H, Silva LHE, Camargo E. High prevalence of Asymptomatic Plasmodium vivax and Plasmodium falciparum infections in native Amazonian Populations. Am J Trop Med Hyg 2002; 66:641-8.

16. Suárez-Mutis MC, Martínez-Espinosa FE, Albuquerque B, Coura JR. Malária. In: Coura JR, organizador. Dinâmica das doenças infecciosas. Rio de Janeiro: Editora Guanabara Koogan; 2005. p. 833-58.

17. Branch OL, Casapia WM, Gamboa DV, Hernandez JN, Alava FF, Roncal N, et al. Clustered local transmission and asymptomatic Plasmodium falciparum and Plasmodium vivax malaria infections in a recently emerged, hypoendemic Peruvian Amazon community. Malar J 2005; 4:27-43.

Recebido em 17/Jan/2006

Versão final reapresentada em 27/Jul/2006

Aprovado em 25/Ago/2006 